\title{
Efeito do sistema de aquecimento hidráulico na temperatura corporal de cadelas durante a recuperação anestésica
}

\author{
Effect of hydraulic heating system on body temperature of female dogs during anesthetic recovery \\ Efecto del sistema de calentamiento hidráulico sobre la temperatura corporal de las perras durante
} la recuperación anestésica

\section{Resumo}

Ovariohisterectomia é um procedimento importante tanto para a saúde e o bem-estar animal quanto para a sociedade. No entanto, há durante o processo uma predisposição do animal a hipotermia, decorrente do procedimento anestésico e cirúrgico. Para estudar o efeito do piso radiante hidráulico na temperatura corporal de cadelas durante a recuperação anestésica foram observados 130 animais submetidos à ovariohisterectomia, provenientes do Centro de Castração e Identificação Animal de Araquari (CCIAA). Avaliaram-se parâmetros fisiológicos, tais como coloração da mucosa oral, temperatura retal, frequência respiratória e cardíaca em animais alojados em baia com piso radiante hidráulico (CP) e baia com cobertor (SP). As avaliações ocorreram entre o período de entrada do animal (T0) no CCIAA até o seu retorno ao lar (T4). Para análises da relação dos parâmetros fisiológicos com o desenvolvimento físico dos animais foram criados dois subgrupos: um baseado no porte (pequeno, médio e grande) e outro na idade (jovens e adultos). A relação entre os parâmetros fisiológicos e temperatura ambiente foi estudada dividindo-se o período experimental em dias frios, moderados e quentes. Os resultados foram submetidos às análises de qui-quadrado e variância para dados não normais. As análises foram feitas ao nível de 5\% de probabilidade de erro. O piso radiante hidráulico mostrou-se eficiente na recuperação anestésica dos animais, independente de porte e idade. Além disso, observou-se maior necessidade do sistema de aquecimento ativo em dias com temperaturas baixas. Concluímos que o piso radiante hidráulico é uma medida eficaz e segura de aquecimento para prevenção da hipotermia no pósoperatório.

Palavras-chave: Bem-estar animal; Centro de castração; Controle de temperatura; Hipotermia; Ovariohisterectomia.

\begin{abstract}
Ovariohysterectomy is an important procedure for health and walfare animal, even as for society. However, during the procedure there is a predisposition of the animal to hypothermia, due to the anesthesia and surgery. In order to study the effect of hydraulic radiant floor on the body temperature of female dogs submitted to ovaryiohysterectomy, 130 animals, coming from the Araquari Animal Castration and Identification Center (CCIAA), were evaluated. Physiological parameters were collected, such as color of the oral mucosa, rectal temperature, respiratory and heart rate in animals housed in pen with hydraulic radiant floor $(\mathrm{CP})$ and blanket $(\mathrm{SP})$. The evaluations took place between the period of entry of the animal (T0) in the CCIAA until its return to home (T4). To analyze the relationship between the physiological parameters and the physical development of the animal, subgroups were created: one based on size (small, medium and large) and other on age (young and adults). The relationship between physiological parameters and room temperature was studied by dividing the experimental period into cold, moderate and warm days. The results were subjected to chi-square and variance analysis for non-normal data. The analyzes were made at the level of $5 \%$ probability of error. The hydraulic radiant floor proved to be efficient in anesthetic recovery of animals, regardless
\end{abstract}


of the size and age. In addition, there was a greater need for the heating system on days with low temperatures. We conclude that the hydraulic radiant floor is an effective and safe way for heating and to prevent hypothermia in the postoperative period.

Keywords: Animal welfare; Castration center; Temperature control; Hypothermia; Ovariohysterectomy.

\section{Resumen}

Ovariohisterectomía es un procedimiento importante tanto para la salud y el bienestar de los animales como para la sociedad. Sin embargo, durante el proceso existe una predisposición del animal a la hipotermia, resultante del procedimiento anestesico y quirúrgico. Para estudiar el efecto del suelo radiante hidráulico sobre la temperatura corporal de las perras durante la recuperación anestésica, se observaron 130 animales, del Centro de Identificación y Castración de Animales de Araquari (CCIAA), sometidos a ovariohisterectomía. Se evaluaron parámetros fisiológicos, como color de la mucosa oral, temperatura rectal, frecuencia respiratoria y cardíaca en animales alojados en corral con calefacción por suelo radiante (CP) corral con manta (SP). Las evaluaciones se realizaron entre el período de ingresso del animal (T0) en la CCIAA hasta su regreso a casa (T4). Para analizar la relación de los parámetros fisiológicos con el desarrollo fisico de los animales, se crearon dos subgrupos: uno por tamaño (pequeño, mediaano y grande) y otro por edad (joven y adulto). La relación entre los parámetros fisiológicos y la temperatura ambiente se estudió dividiendo el período experimental en días fríos, moderados y calurosos. Los resultados se sometieron a análisis de chi-cuadrado y varianza para datos no normales. Los análisisis se realizaron con un 5\% de probabilidad de error. La calefacción por suelo radiante hidráulico demostró ser eficaz en la recuperación anestésica de los animales, independientemente de su tamaño y edad. Además, existía una mayor necesidad del sistema de calefacción activa en los días con bajas temperaturas. Concluimos que la calefacción por suelo radiante hidráulica es una medida de calefacción eficaz y segura para prevenir la hipotermia posoperatoria.

Palabras clave: Bienestar de los animales; Centro de castracion; Control de temperatura; Hipotermia; Ovariohisterectom.

\section{Introdução}

O controle populacional de cães é uma questão de saúde pública e o aumento descontrolado desses animais amplia os casos de acidentes em vias públicas e as possibilidades de transmissões de zoonoses (Luz, Münnich, \& Vannucchi, 2015). Além disso, famílias de baixa renda apresentam dificuldades para alimentar e esterilizar os animais acolhidos (Oliveira Filho, Batista, \& Santos, 2020). Neste contexto, a ovariohisterectomia (OH) realizada por instituições públicas, como Centros de Castração Animal, constitui uma forma viável de evitar a procriação desenfreada (Annandale, Scheepers, \& Fosgate, 2020), principalmente em animais de abrigos ou pertencentes à população socioeconômica carente. O Centro de Castração e Identificação Animal (CCIAA) tem como intuito consciencializar a população do problema relativo ao abandono, maus-tratos de animais e superpopulação de animais abandonados, bem como, reforçar os conceitos de guarda responsável, além de realizar castrações gratuitas.

Além de atuar no controle populacional e favorecer o bem-estar animal, a esterilização de cadelas possui diversas vantagens, (Brunna Fernanda Arraez Alves, 2020) dentre elas a prevenção de desenvolvimento de neoplasias mamárias (Root Kustritz, 2014), doenças ovarianas, tais como tumores e cistos ovarianos (Van Goethem, Schaefers-Okkens, \& Kirpensteijn, 2006), piometra ( Van Goethem et al., 2006; Root Kustritz, 2014), hiperplasia e prolapso vaginal (Root Kustritz, 2014), gestações indesejadas, complicações, aborto, distocia, prolapso uterino e subinvolução placentária (Viana, 2014). A OH também auxilia na estabilização de doenças não relacionadas ao sistema reprodutor, tais como diabetes, epilepsia e sarna demodécica generalizada (Andrade \& Bittencourt, 2013).

Uma das principais preocupações relacionadas à um procedimento anestésico, que é indispensável para a realização da $\mathrm{OH}$, é a ocorrência de hipotermia. Problemas de termorregulação em cães apresentam como principais sinais clínicos: tremores; variação da cor da mucosa oral; membros (como orelhas, patas e cauda) gelados; respiração lenta e pulso fraco; letargia comportamental; e temperatura retal baixa (Clark-Price, 2015; Junior, 2015).

$\mathrm{O}$ uso de técnicas para reduzir a hipotermia pode melhorar o atendimento ao paciente e amenizar os efeitos colaterais associados à redução da temperatura corporal. Em pacientes caninos, é recomendado essa intervenção para períodos anestésicos superiores a 20 minutos (Clark-Price, 2015). Tais técnicas são classificadas em passiva, ativa interna (metabólica) 
ou ativa externa. Passivas são aquelas que utilizam materiais isolantes térmicos para cobrir o paciente (Pottie, Dart, Perkins, \& Hodgson, 2007; Clark-Price, 2015). Essas técnicas reduzem a perda de calor por convecção e reaproveitam o calor perdido por radiação (Pelzer et al., 2017). As técnicas metabólicas induzem o corpo a produzir maiores quantidades de calor endógeno por meio da manipulação do metabolismo (Clark-Price, 2015). Técnicas ativas incluem uma fonte externa de calor, responsável por minimizar o gradiente de temperatura entre o corpo do paciente e o meio externo (Pottie et al., 2007). São exemplos de dispositivos utilizados nessa técnica os cobertores elétricos, as lâmpadas quentes, os aquecedores de fluidos intravenosos, almofadas de circulação de fluido quente e pisos térmicos.

Ao reduzir a perda de calor contribui-se para diminuir os efeitos colaterais associados a hipotermia (Gide, 1967;

Biazzotto, Brudniewski, Schmidt, \& Costa Auler Jr., 2006). É importante, no entanto, tomar todos os cuidados para que utilizando-se uma fonte de calor, ela não ofereça risco de queimaduras ao animal (Alves, 2007). Com esta finalidade, foi desenvolvido um piso radiante hidráulico com estrutura adaptada para a realidade do Centro de Castração e Identificação Animal de Araquari (CCIAA), para ser aplicado em procedimentos operatórios em animais de rua ou pertencentes à munícipes de baixa renda.

Desse modo, o objetivo deste trabalho é avaliar a eficiência do piso radiante hidráulico no combate à hipotermia, estudando seus efeitos nos parâmetros fisiológicos de cadelas submetidas a $\mathrm{OH}$ eletiva.

\section{Meterial e Métodos}

Os procedimentos realizados nesta pesquisa foram aprovados pelo Comitê de Ética no Uso de Animal (CEUA) do Instituto Federal Catarinense (IFC), Campus Araquari, sob o protocolo n ${ }^{\circ} 340 / 2020$ e autorizados pelos proprietários mediante a assinatura do Termo de Consentimento Livre e Esclarecido (TCLE). Para elaboração e organização estrutural desta pesquisa usamos como base Fontelles M. J. et el. (2009), com o auxílio das etapas de maneira sucintas, no planejamento e desenvolvimento do método escolhido, a colteta e a tabulação dos dados até a divulgação dos resultados e a elaboração da conclusão.

O trabalho foi desenvolvido entre os meses de junho e outubro de 2020. Neste período foram submetidas à cirurgia de ovariohisterectomia 130 cadelas, consideradas saudáveis em exame físico, sem raça definida (SRD), com idade e peso corporal variável, sendo, por isso, divididas em: jovem (0 a 2 anos) e adultos (3 a 8 anos), de acordo com (Creevy et al., 2019); pequenos $(<10 \mathrm{~kg}$ ), médios (10-22 kg) e grandes ( $>22 \mathrm{~kg}$ ), de acordo com Medina (2018). Todos os animais estudados são provenientes da rotina cirúrgica do Centro de Castração e Identificação Animal de Araquari (CCIAA). O CCIAA faz parte de um projeto da Fundação Municipal do Meio Ambiente de Araquari (FUNDEMA) e está localizado a latitude 26²2'12" sul e longitude 48 43'20" oeste, com altitude de 9 metros (IBGE, 2019). Trata-se de uma região mesotérmica úmida com verão quente (Cfa), segundo a classificação de Köppen-Geiger (Geiger, 2021).

$\mathrm{O}$ método aplicado nesta pesquisa foi definido através do protocolo diário da rotina do Centro de Castração e Identificação Animal de Araquari (CCIAA), não havendo intervenção na sua prática de esterilização. Baseado no procedimento cirúrgico, foi implantado o piso radiante hidráulico para auxiliar no pós-operatório das cadelas submetidas à ovariohisterectomia. A estrutura do piso radiante foi desenvolvida por quatro camadas, sendo a primeira um isolante térmico (isopor), a segunda formada por tubulação (mangueiras de cristal transparente), seguida de uma rede metálica de sustentação e, por fim, a argamassa (Rohr et al., 2019). Em um reservatório de água foram acoplados resistores para aquecimento da água e bomba mecânica para circulação do fluido aquecido pelo piso. Um termostato foi ligado ao piso para mantê-lo numa temperatura próxima dos $40^{\circ} \mathrm{C}$. 
Antes do procedimento cirúrgico solicitou-se jejum hídrico e alimentar de no mínimo 8 e 12 horas, respectivamente. Chegando ao centro de castração as cadelas passaram por triagem e realizou-se a avaliação dos parâmetros fisiológicos (basal), tais como:

i) Avaliação da mucosa: Subjetiva, realizada por observação visual da coloração da mucosa oral. Classificada em corada, pálida ou cianótica;

ii) Temperatura Retal $\left({ }^{\circ} \mathrm{C}\right.$ ): Coletada com termômetro clínico digital (G-Tech, modelo TH150), com faixa de temperatura entre $32,0-42,9^{\circ} \mathrm{C}$. O mesmo foi mantido em contato direto com a mucosa retal durante a mensuração;

iii) Frequência Respiratória (FR): Obtida através da observação dos movimentos torácicos, em movimentos por minuto (mpm);

iv) Frequência Cardíaca (FC): Obtida em batimentos por minuto (bpm), utilizando-se um estetoscópio cardiológico (Premium).

Aferiu-se o peso dos animais em balança digital (300 kg - 60x80 Micheletti), anotou-se a idade e, em seguida, as cadelas foram alojadas individualmente em baias de alvenaria. A temperatura da sala do pré-operatório foi avaliada com termômetro de bulbo seco de mercúrio analógico e a temperatura do piso foi mantida em aproximadamente $40^{\circ} \mathrm{C}$, com auxílio de um termostato digital W1209. Os dias experimentais foram classificados em frio $\left(<18^{\circ} \mathrm{C}\right)$, moderado $\left(19-22^{\circ} \mathrm{C}\right)$ e quente $\left(>23^{\circ} \mathrm{C}\right)$.

Para melhor compreensão do processo, a descrição metodológica foi dividida em etapas, que serão apresentadas a seguir.

\subsection{Pré-operatório}

Todos os pacientes foram submetidos, ao mesmo protocolo anestésico estabelecido pelo CCIAA. A medicação préanestésica foi composta pela associação de morfina $(0,5 \mathrm{mg} / \mathrm{kg}$, IM) e xilazina $2 \%(1,8 \mathrm{mg} / \mathrm{kg}$, IM) e a indução da anestesia foi realizada com cetamina $10 \%(9 \mathrm{mg} / \mathrm{kg}$, IM). Posteriormente, realizou-se tricotomia e antissepsia da face cranial do antebraço para realização de venopunção cefálica com cateter de tamanho compatível com o porte do animal, para administração de cloreto de sódio $0,9 \%$. Na sequência, conduziu-se o animal para o centro cirúrgico.

\subsection{Transoperatório}

Durante o procedimento cirúrgico a temperatura da sala foi mantida entre 21 e $23^{\circ} \mathrm{C}$. Previamente ao início da cirurgia administrava-se um bolus de diazepam $(0,5 \mathrm{mg} / \mathrm{kg}$, IV) e, na sequência, iniciava-se o procedimento de ovariohisterectomia. Quando a cadela demonstrava sinais de superficialização anestésica, tais como reflexo visual, agitação e respiração ofegante, a anestesia era complementada utilizando-se a combinação de cetamina $10 \%$ e xilazina $2 \%$.

Todos os procedimentos cirúrgicos foram realizados pelo mesmo cirurgião e auxiliar. O tempo médio do período transoperatório foi em torno de 40 minutos.

\subsection{Pós-operatório}

No pós-operatório foram administrados cetoprofeno (1 mg/kg, IV), dipirona sódica (25 mg/kg, IV), tramadol (2,5 $\mathrm{mg} / \mathrm{kg}$, IV) e enrofloxacino ( $5 \mathrm{mg} / \mathrm{kg}$, IV). Por fim, os animais receberam a identificação através de microchip.

Após os procedimentos cirúrgicos, cada cadela foi alocada em baia individual. Os pacientes foram divididos em dois grupos de observação, de acordo com o sistema de aquecimento disponível nas baias: i) Com Piso (CP), alojados em baias com o sistema de aquecimento ativo (piso radiante hidráulico) e passivo (coberta); e ii) Sem Piso (SP), contando apenas com sistema de aquecimento passivo (coberta). 
Para a sistematização da coleta de dados utilizou-se cinco momentos: i) T0 - Entrada da cadela no CCIAA; ii) T1 Entrada na sala de cirurgia; iii) T2 - Final da cirurgia e entrada na sala de recuperação; iv) T3 - Trinta minutos após a cirurgia; e v) T4 - Tempo necessário para o paciente atingir a temperatura ideal, definida em $36,7^{\circ} \mathrm{C}$ (Grubb et al., 2020). Ressalta-se, aqui, que houveram animais que não atingiram a temperatura ideal no tempo de observação. Por isso, nesses casos, T4 foi definido como o tempo de permanência do animal no CCIAA.

\section{Análise de Resultados}

Para analisar a relação entre a coloração da mucosa e as variáveis classificatórias (piso, peso, idade, temperatura ambiente) e os momentos experimentais os dados foram submetidos às análises de qui-quadrado (PROC FREQ). Os efeitos das variáveis classificatórias e os momentos experimentais sobre a frequência cardíaca, frequência respiratória e temperatura retal foram avaliados pela análise de variância para dados não normais (PROC NPAR1WAY). Os dados foram analisados ao nível de 5\% de probabilidade de erro ( $\mathrm{P}<0,05)$ usando o programa SAS (Analysis System Institute, Cary, NC, USA, versão 9.3). Os resultados de Frequência Cardíaca e Respiratória foram expressos por média \pm desvio padrão médio.

\section{Resultados e Discussão}

$\mathrm{Na}$ chegada dos animais ao CCIAA (T0) os dados fisiológicos coletados demonstram homogeneidade entre os grupos, uma vez que não houve diferença significativa $(\mathrm{P}>0,05)$ para a coloração da mucosa oral, temperatura retal, frequências cardíaca e respiratória (Tabela 1). 
Tabela 1 - Comparação dos parâmetros fisiológicos das cadelas $(\mathrm{n}=130)$ do grupo com piso $(\mathrm{CP}, \mathrm{n}=65)$ e sem piso $(\mathrm{SP}, \mathrm{n}=$ 65) nos diferentes momentos avaliados. Da entrada a saída do CCIAA (N); chegada do animal (T0); na mesa de cirurgia (T1); no fim da cirurgia (T2); meia hora após a cirurgia (T3); e quando a cadela recebe alta (T4). Temperatura retal ideal estabelecida em $36,7^{\circ} \mathrm{C}$ (Grubb et al., 2020).

\begin{tabular}{|c|c|c|c|c|c|}
\hline \multicolumn{6}{|c|}{ COLORAÇÃO DA MUCOSA (\%) } \\
\hline & \multicolumn{2}{|c|}{$\mathbf{C P}$} & \multicolumn{2}{|c|}{ SP } & \multirow[b]{2}{*}{$\mathbf{P}>\mathbf{F}$} \\
\hline & Corada & Pálida & Corada & Pálida & \\
\hline $\mathbf{N}$ & 90,15 & 9,85 & 84,62 & 15,38 & 0,0335 \\
\hline T0 & 100 & 0 & 98,46 & 1,54 & 0,3154 \\
\hline T1 & 98,46 & 1,54 & 96,92 & 3,08 & 0,5591 \\
\hline $\mathbf{T 2}$ & 80 & 20 & 75,38 & 24,62 & 0,5274 \\
\hline T3 & 75,38 & 24,62 & 72,31 & 27,69 & 0,6898 \\
\hline T4 & 96,92 & 3,08 & 80 & 20 & $0,0025^{*}$ \\
\hline \multicolumn{6}{|c|}{ TEMPERATURA RETAL MÉDIA (\%) } \\
\hline & \multicolumn{2}{|c|}{$\mathbf{C P}$} & \multicolumn{2}{|c|}{ SP } & \\
\hline & Atingiu & Não & Atingiu & Não & $\mathbf{P}>\mathbf{F}$ \\
\hline $\mathbf{N}$ & 96,92 & 3,08 & 86,15 & 13,85 & $0,0001 *$ \\
\hline \multicolumn{6}{|c|}{ FREQUÊNCIA CARDÍACA (bpm) } \\
\hline & \multicolumn{2}{|c|}{$\mathbf{C P}$} & \multicolumn{2}{|c|}{ SP } & $\mathbf{P}>\mathbf{F}$ \\
\hline T0 & \multicolumn{2}{|c|}{$103,02 \pm 16,66$} & \multicolumn{2}{|c|}{$100,37 \pm 17,04$} & 0,5193 \\
\hline T1 & \multicolumn{2}{|c|}{$89,47 \pm 17,10$} & \multicolumn{2}{|c|}{$91,94 \pm 15,09$} & 0,4896 \\
\hline $\mathbf{T} 2$ & \multicolumn{2}{|c|}{$72,06 \pm 11,52$} & \multicolumn{2}{|c|}{$73,82 \pm 12,72$} & 0,4979 \\
\hline T3 & \multicolumn{2}{|c|}{$74,71 \pm 13,42$} & \multicolumn{2}{|c|}{$71,42 \pm 13,93$} & 0,2672 \\
\hline T4 & \multicolumn{2}{|c|}{$81,29 \pm 12,56$} & \multicolumn{2}{|c|}{$80,62 \pm 13,57$} & 0,8018 \\
\hline \multicolumn{6}{|c|}{ FREQUÊNCIA RESPIRATÓRIA (mpm) } \\
\hline & \multicolumn{2}{|c|}{$\mathbf{C P}$} & \multicolumn{2}{|c|}{ SP } & $\mathbf{P}>\mathbf{F}$ \\
\hline T0 & \multicolumn{2}{|c|}{$25,56 \pm 4,17$} & \multicolumn{2}{|c|}{$25,97 \pm 5,56$} & 0,6917 \\
\hline T1 & \multicolumn{2}{|c|}{$20,92 \pm 5,07$} & \multicolumn{2}{|c|}{$20,60 \pm 5,26$} & 0,7993 \\
\hline $\mathbf{T} 2$ & \multicolumn{2}{|c|}{$25,17 \pm 6,67$} & \multicolumn{2}{|c|}{$24,32 \pm 6,62$} & 0,5634 \\
\hline T3 & \multicolumn{2}{|c|}{$27,51 \pm 6,90$} & \multicolumn{2}{|c|}{$24,83 \pm 5,56$} & $0,0597 *$ \\
\hline T4 & \multicolumn{2}{|c|}{$30,43 \pm 6,93$} & \multicolumn{2}{|c|}{$25,28 \pm 5,59$} & $0,0012 *$ \\
\hline
\end{tabular}

*Diferença estatística. Fontes: Autores (2021).

Ressalta-se que não foi encontrado animal com a coloração da mucosa cianótica na amostra experimental estudada. Nota-se, entretanto, tendência de redução do número de animais com mucosa corada do início da cirurgia (T1) até meia hora após seu término (T3), sem diferença estatística evidente. Porém, no momento em que as cadelas recebem alta (T4), houve aumento significativo $(\mathrm{P}=0,0025)$ do número de animais apresentando mucosa corada no grupo $\mathrm{CP}(\mathrm{Tabela} 1)$. A análise estatística geral $(\mathrm{N})$, realizada com os dados de coloração de mucosa do momento da entrada até a saída do animal, também mostraram diferença significativa entre os grupos $(\mathrm{P}=0,03)$ (Tabela 1). Desta forma, o uso do piso radiante hidráulico mostrou-se efetivo para manter a coloração da mucosa oral corada.

Logo após o término do procedimento cirúrgico (T2), os animais pertencentes ao grupo CP foram colocados sobre o piso radiante hidráulico. Seguindo recomendações, o piso radiante hidráulico permaneceu ligado até que o paciente apresentasse temperatura retal próxima ao valor ideal, previamente definida em $36,7^{\circ} \mathrm{C}$ (Grubb et al., 2020). Essa temperatura 
é suficiente para que sejam restauradas as funções cardiovasculares e a coagulação sanguínea, sem sobrecarregar o sistema circulatório, além de ajudar a prevenir o fenômeno afterdrop, através da redução do gradiente de temperatura centro-periferia (Root Kustritz, 2014; Grubb et al., 2020).

Em relação a temperatura retal média, os resultados mostram que houve diferença significativa entre os grupos $(\mathrm{P}=$ 0,0001), sendo que quase todos os animais alojados na baia com o piso radiante hidráulico (CP) permaneceram acima da temperatura ideal durante o período avaliado, enquanto que pouco menos de $87 \%$ dos animais alojados em baia com cobertor conseguiram resultado semelhante (Tabela 1).

A diminuição da temperatura corporal de cães para valores abaixo de $36,7^{\circ} \mathrm{C}$ pode resultar em efeitos adversos, tais como o retardamento do metabolismo, a disfunção cardiovascular, prejuízos a perfusão, o comprometimento respiratório, a depressão cerebral e o aumento da incidência de infecções (Radlinsky, 2015; Grubb et al., 2020). Além disso, letargia metabólica induzida por drogas pode resultar em dificuldades de recuperação, prolongando o processo (Pottie et al., 2007).

Em relação a Frequência Cardíaca (FC) não foram observadas diferenças significativas entre os grupos ( $\mathrm{P}>0,05)$, havendo tendência de redução de FC entre o momento da chegada do animal ao centro (T0) até meia hora depois da cirurgia (T3). Em seguida, observa-se um leve aumento. Acredita-se que tal comportamento esteja relacionado aos efeitos da anestesia. (Barcelos et al., 2021; Pelzer et al., 2017)

A Frequência Respiratória (FR) dos animais na chegada ao CCIAA era de aproximadamente $26 \mathrm{mpm}$ e aumentou significativamente $(\mathrm{P}=0,0012)$ nos animais do grupo $\mathrm{CP}$ (acima de $30 \mathrm{mpm}$ ) no momento em que eles se preparavam para retornar aos lares (T4). Tal fato pode estar relacionado ao aumento da temperatura retal média, uma vez que um mecanismo importante utilizado pelos cães na troca de calor com o ambiente é a respiração (English et al., 1990). O fato dos cães do grupo CP já terem atingido a temperatura ideal explica a capacidade de manter funções mecânicas essenciais para a respiração e a FR alta, facilitando a liberação de calor extra (Howe, 2006).

A Figura 1 mostra a variação da temperatura retal média de cadelas de diferentes portes nos diferentes momentos avaliados, entre os grupos CP e SP. 
Figura 1 - Temperatura Retal Média nos diferentes momentos experimentais para os diferentes portes de cadelas: a) Pequena; b) Média; e c) Grande. As avaliações foram realizadas para animais no sistema com piso radiante hidráulico (CP) e com cobertor (SP). A linha tracejada vertical indica o momento em que as cadelas foram colocadas sobre o piso radiante hidráulico. Letras maiúsculas diferentes expressam diferença significativa entre os grupos (com piso - CP e sem piso - SP) em cada momento experimental $(\mathrm{P}<0,05)$. Letras minúsculas diferentes expressam diferença significativa dentro de cada grupo (com piso - CP) e (sem piso - SP) em cada momento experimental $(\mathrm{P}<0,05)$.

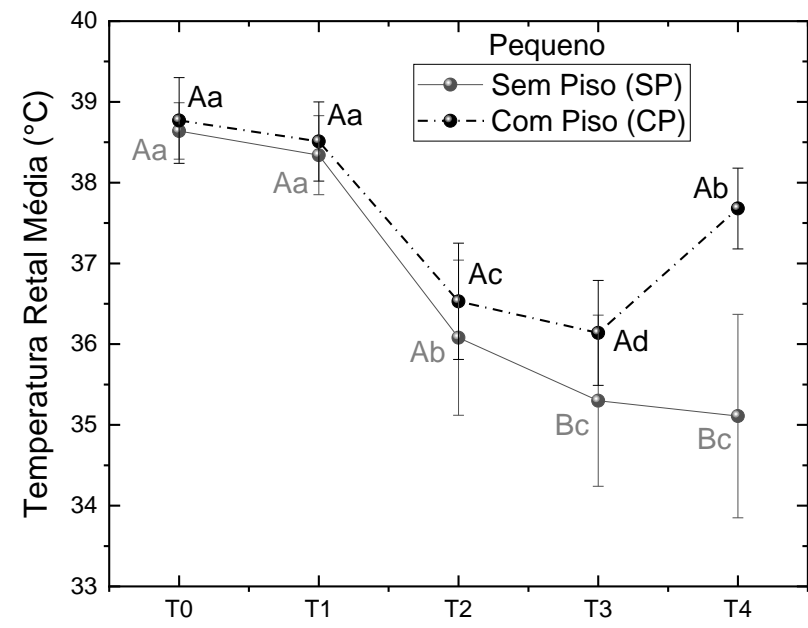

a)

Momentos Experimentais

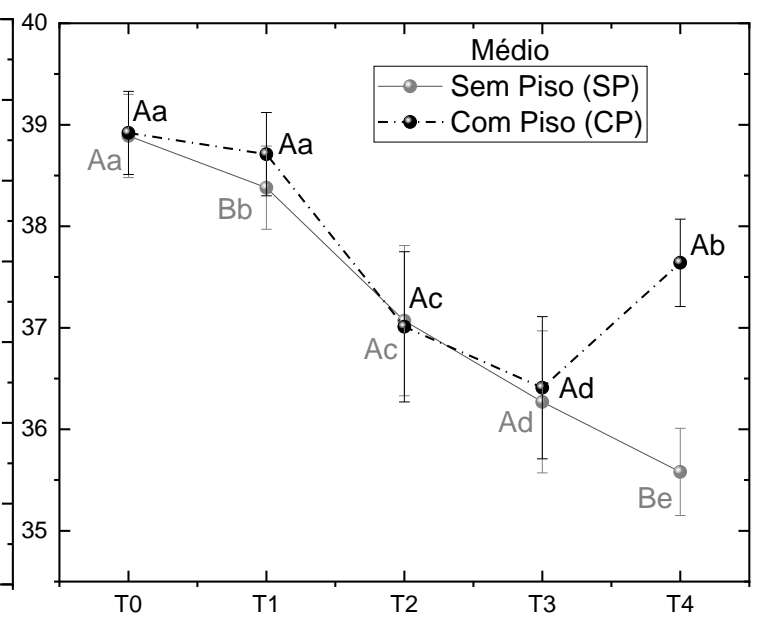

b) Momentos Experimentais

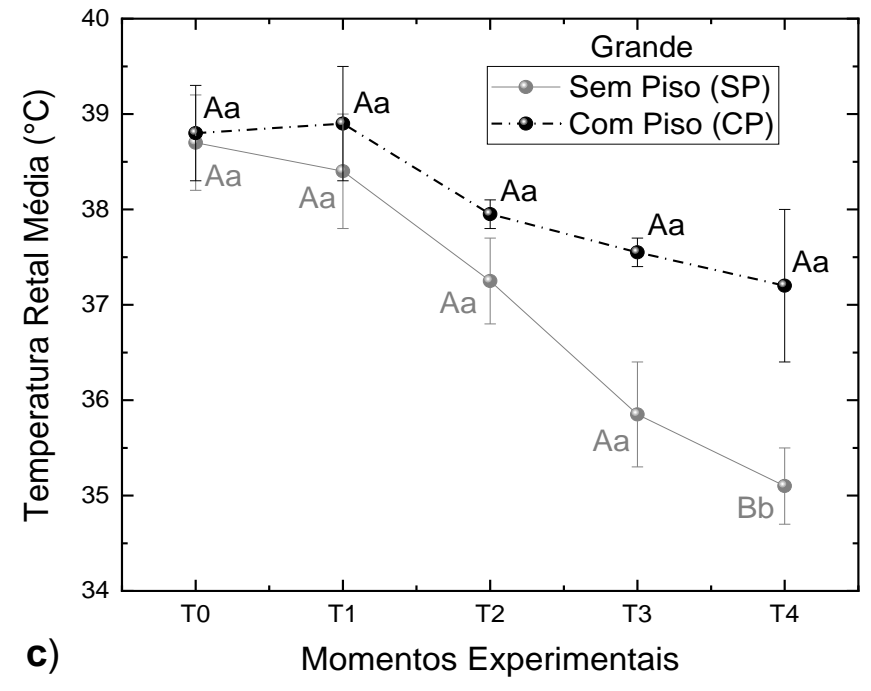

Fonte: Autores (2021).

Observa-se que, independentemente do porte (pequeno, médio ou grande), o grupo SP, durante todo o período avaliado, apresenta queda da temperatura retal média. Após os animais serem colocados sobre o piso radiante hidráulico (T2), nota-se aumento significativo da temperatura retal média para animais pequenos em T3 $(\mathrm{P}=0,0001)$ e T4 ( $\mathrm{P}=0,0001)$ (Figura 1a) e para animais de médio e grande porte em T4 ( $\mathrm{P}=0,0001$ e $\mathrm{P}=0,0213$, respectivamente) (Figura 1b e 1c). Sabe-se que animais de pequeno porte estão mais propensos a hipotermia, pois perdem mais calor por irradiação (Pelzer et al., 2017). Desta forma, o emprego do piso radiante hidráulico mostrou-se um importante instrumento para o reestabelecimento da temperatura corporal dos animais, independente dos seus tamanhos corporais.

Nas três primeiras horas de anestesia, cães normalmente apresentam uma queda da temperatura corporal de aproximadamente $3^{\circ} \mathrm{C}$ (Goleman, Daniel; Boyatzis, Richard; Mckee, 2019), sendo que a redução de temperatura induzida por anestesia pode ser dividida em 3 fases: i) logo após a indução, com rápida redução da temperatura central pela redistribuição do calor para a periferia; ii) em seguida a temperatura diminui linearmente com o tempo, pois a taxa de produção metabólica é 
menor que a perda de calor para o ambiente; iii) depois disso a vasoconstrição restringe o fluxo de calor da região central para a periferia do animal. Nesse momento, o paciente atinge um segundo patamar de equilíbrio térmico, com temperatura abaixo do valor basal (Rodrigues, Quessada, Moraes, Dantas, \& Sales, 2017).

Neste trabalho, para o grupo de animais SP, encontrou-se variações de temperatura retal média entre T0 e T4 de $3,53^{\circ} \mathrm{C}$ em animais pequenos, de $3,31^{\circ} \mathrm{C}$ em animais médios e $3,60^{\circ} \mathrm{C}$ em animais grandes. Os resultados dessa pesquisa estão de acordo com o relatado para animais alojados em baias com sistema de aquecimento passivo (Redondo et al., 2012). Já os animais da baia com o piso radiante hidráulico (CP) tiveram variações de temperatura retal de $1,09^{\circ} \mathrm{C}$ no grupo de pequenos, $1,28^{\circ} \mathrm{C}$ no grupo de médios e $1,60^{\circ} \mathrm{C}$ no grupo de grandes. Em média, a variação de temperatura retal para animais que usufruíram do piso foi de pouco mais de $1{ }^{\circ} \mathrm{C}\left(1,33^{\circ} \mathrm{C}\right)$, que está bem abaixo da variação de temperatura retal média dos animais que não tiveram esse recurso $\left(3,48^{\circ} \mathrm{C}\right)$.

Da análise de variação da temperatura retal média, entre os diferentes portes, observa-se uma resposta mais rápida dos animais menores (Figura 1a) em relação aos maiores (Figura 1c). Isso ocorre porque as trocas de calor entre animais pequenos e o ambiente são mais rápidas, devido ao menor gradiente de temperatura entre a parte central do animal e a sua periferia (Redondo et al., 2012; Potter et al., 2015).

A Tabela 2 apresenta os parâmetros fisiológicos das cadelas divididas em grupo, de acordo com o seu porte e idade, considerando a temperatura ambiente no dia da coleta. As análises da relação da mucosa e da temperatura retal média entre os grupos divididos por porte indicaram que não há diferença significativa entre os grupos (Tabela 2). Da mesma forma, os grupos jovens e adultos também não apresentaram diferença significativa em relação a coloração da mucosa (Tabela 2). Isso implica que, na recuperação anestésica, a variação da coloração da mucosa é independente do porte e da idade das cadelas.

Tabela 2 - Comparação dos parâmetros fisiológicos das cadelas $(n=130)$ dos grupos divididos por porte (pequeno, médio, grande) e idade (jovem e adulto). Considera-se também três classes de temperatura ambiente: quente, moderado e frio.

\begin{tabular}{|c|c|c|c|c|c|c|c|}
\hline \multicolumn{8}{|c|}{ COLORAÇÃO DA MUCOSA (\%) } \\
\hline & \multicolumn{2}{|c|}{ Pequeno } & \multicolumn{2}{|c|}{ Médio } & \multicolumn{2}{|c|}{ Grande } & \multirow[b]{2}{*}{$\mathbf{P}>\mathbf{F}$} \\
\hline & Corada & Pálida & Corada & Pálida & Corada & Pálida & \\
\hline \multirow[t]{3}{*}{$\mathbf{N}$} & 87,3 & 12,7 & 87,62 & 12,38 & 85 & 15 & 0,9417 \\
\hline & \multicolumn{2}{|c|}{ Jovens } & \multicolumn{2}{|c|}{ Adultos } & & & \\
\hline & Corada & Pálida & Corada & Pálida & & & $\mathbf{P}>\mathbf{F}$ \\
\hline \multirow[t]{3}{*}{$\mathbf{N}$} & 88,47 & 11,53 & 85,33 & 14,67 & & & 0,2518 \\
\hline & \multicolumn{2}{|c|}{ Quente } & \multicolumn{2}{|c|}{ Moderado } & \multicolumn{2}{|c|}{ Frio } & \\
\hline & Corada & Pálida & Corada & Pálida & Corada & Pálida & $\mathbf{P}>\mathbf{F}$ \\
\hline $\mathbf{N}$ & 92,21 & 7,32 & 89,34 & 10,66 & 78,03 & 21,97 & 0,0011 \\
\hline & \multicolumn{7}{|c|}{ TEMPERATURA RETAL MÉDIA $\left({ }^{\circ} \mathrm{C}\right)$} \\
\hline & \multicolumn{2}{|c|}{ Pequeno } & \multicolumn{2}{|c|}{ Médio } & \multicolumn{2}{|c|}{ Grande } & $\mathbf{P}>\mathbf{F}$ \\
\hline $\mathbf{N}$ & \multicolumn{2}{|c|}{37,22} & \multicolumn{2}{|c|}{37,47} & \multicolumn{2}{|c|}{37,57} & 0,0866 \\
\hline & \multicolumn{2}{|c|}{ Jovens } & \multicolumn{2}{|c|}{ Adultos } & & & $\mathbf{P}>\mathbf{F}$ \\
\hline $\mathbf{N}$ & \multicolumn{2}{|c|}{37,36} & \multicolumn{2}{|c|}{37,33} & \multirow{2}{*}{\multicolumn{2}{|c|}{ Frio }} & 0,7745 \\
\hline & \multicolumn{2}{|c|}{ Quente } & \multicolumn{2}{|c|}{ Moderado } & & & $\mathbf{P}>\mathbf{F}$ \\
\hline $\mathbf{N}$ & \multicolumn{2}{|c|}{37,04} & \multicolumn{2}{|c|}{37,45} & \multicolumn{2}{|c|}{3719} & 0,0253 \\
\hline
\end{tabular}

Da entrada a saída da clínica (N); Temperatura retal ideal estabelecida em $36,7^{\circ} \mathrm{C}$ (Grubb et al., 2020). Fonte: Autores (2021). 
As análises da relação da temperatura retal média entre os grupos divididos por porte e idade indicaram que não há diferença significativa entre os grupos (Tabela 2). Este resultado implica que a variação de temperatura retal média de cadelas em procedimento anestésico é independente de porte ou idade.

Embora a idade não seja diretamente um problema em procedimentos anestésicos, as patologias ocorrem com mais frequência em pacientes idosos. Já os pacientes neonatais e pediátricos podem apresentar sistemas fisiológicos imaturos e encontrarão maiores dificuldades de respostas em condições anestésicas (Grubb et al., 2020). Cães em idade avançada ou muito jovens podem ter o risco aumentado pela indução anestésica devido a deficiências naturais dos sistemas cardiovascular, respiratório, renal, hepático e neurológico, que podem torná-los incapazes de responder eficazmente a hipotensão ou hipotermia causados pela anestesia (Brodbelt et al., 2007; Grubb et al., 2020).

Já em relação a temperatura ambiente foi apresentada diferença significativa $(P=0,0011)$, com melhor resultado para os experimentos realizados em dias quentes, com mais de $92 \%$ dos animais com mucosa corada, e pior resultado em dias frios, com mais de $21 \%$ dos animais com mucosa pálida. Sabe-se que ambientes com temperaturas abaixo de $21^{\circ} \mathrm{C}$ favorecem à hipotermia (João Moreira da Costa Neto, Ricardo Andrés Ramírez Uscátegui, Rodrigo Lima Carneiro, Pedro Isidro da Nóbrega, 2011) e que uma temperatura ambiente acima de $23^{\circ} \mathrm{C}$ pode ajudar a manter a normotermia durante a anestesia (Biazzotto et al., 2006). Neste estudo, a temperatura do centro cirúrgico foi mantida entre 21 e $23^{\circ} \mathrm{C}$, porém, não foi suficiente para manter a temperatura corporal dentro dos limites fisiológicos.

Em relação a temperatura retal média, o porte físico dos animais também não se mostrou relevante para observações de diferenças significativas entre os grupos. Já a variável temperatura ambiente apresentou diferença significativa entre os grupos $(\mathrm{P}=0,0253)$. Observou-se que em dias frios a utilização de uma fonte externa de calor é essencial para menores variações de temperatura corporal de cadelas submetidas à procedimentos cirúrgicos que necessitam de anestesia.

\section{Conclusão}

A utilização do piso radiante hidráulico em sala de recuperação após a ovariohisterectomia contribuiu significativamente para a recuperação anestésica e restabelecimento da temperatura corporal das cadelas, independente de tamanho ou idade. A utilização desse sistema mostra-se ainda mais necessária em dias frios.

O piso radiante hidráulico atuou também como equalizador social, uma vez que garantiu o fornecimento de calor para animais provenientes de famílias pobres, que, em alguns casos, não tem condições de levar cobertas para o Centro de Castração no momento da ovariohisterectomia. $\mathrm{O}$ uso do piso proporcionou também conforto e bem-estar aos animais durante a recuperação anestésica.

O tema permite associar ideias de inovação tecnológica, como a utilização de energia solar para o aquecimento da água. É possível também avaliar a utilização do piso radiante hidráulico já no momento da anestesia até o momento de retorno do animal ao lar. Assim é possível analisar as curvas de variação de temperatura corporal em casos de utilização do piso e sem nenhuma interferência externa no processo de perda de calor devido a anestesia.

\section{Referências}

Alves, B. F. A., \& Hebling, L. M. G. F. (2020). vantagens e desvantagens da castração cirúrgica de cães domésticos. uma revisão integrativa de literatura / advantages and disadvantages of domestic dogs' surgical sterilization. an integrative literature review. Brazilian Journal of Development, 6(9), 73157-73168. https://doi.org/10.34117/bjdv6n9-683

Alves, M. de B. (2007). Prevenção, Controle E Tratamento Da Hipotermia Perioperatória Em Cães. 49. http://www.cirurgia.vet.ufba.br/arquivos/docs/monografias/2007_AlvesMB.pdf

Andrade, ana C. de S., \& Bittencourt, L. H. F. de B. (2013). Castração convencional e precoce: revisão de literatura. Anais Do 11 ${ }^{\circ}$ Encontro Científico Cultural Interinstituicional, (7406), 268-272. Retrieved from http://www.fag.edu.br/upload/ecci/anais/55952ac1cd2cb.pdf 
Annandale, A., Scheepers, E., \& Fosgate, G. T. (2020). The effect of an ovariohysterectomy model practice on surgicaltimes for final-yearveterinary students' first live-animal ovariohysterectomies. Journal of Veterinary Medical Education, 47(1), 44-55. https://doi.org/10.3138/jvme.1217-181r1

Barcelos, L. de C., Tameirão, E. R., Gonzaga, L. W. F., Bastos, L. da S., Oliveira, C. F. A. da, Almeida, J. V. F. C. de, ... Ferrante, M. (2021). Anestesia em pequenos animais durante procedimentos cirúrgicos: Revisão. Pubvet, 15(10), 1-14. https://doi.org/10.31533/pubvet.v15n10a933.1-14

Biazzotto, C. B., Brudniewski, M., Schmidt, A. P., \& Costa Auler Jr., J. O. (2006). Perioperative hypothermia| Hipotermia no período peri-operatório. Revista Brasileira de Anestesiologia, 56(1), 89-106.

Brodbelt, D. C., Pfeiffer, D. U., Young, L. E., \& Wood, J. L. (2007). Risk factors for anaesthetic-related death in cats: results from the confidential enquiry into perioperative small animal fatalities (CEPSAF). British journal of anaesthesia, 99(5), 617-623. https://doi.org/10.1093/bja/aem229)

Brunna F. A. \& Alves, L. M. G. F. H. (2020). Vantagens e desvantagens da castração cirúrgica de cães domésticos. uma revisão integrativa de literatura / advantages and disadvantages of domestic dogs' surgical sterilization. an integrative literature review. Brazilian Journal of Development, 6(9), 73157-73168. https://doi.org/10.34117/bjdv6n9-683

Clark-Price, S. (2015). Inadvertent Perianesthetic Hypothermia in Small Animal Patients. Veterinary Clinics of North America - Small Animal Practice, 45(5), 983-994. https://doi.org/10.1016/j.cvsm.2015.04.005

Creevy, K. E., Grady, J., Little, S. E., Moore, G. E., Strickler, B. G., Thompson, S., \& Webb, J. A. (2019). 2019 AAHA Canine Life Stage Guidelines. Journal of the American Animal Hospital Association, 55(6), 267-290. https://doi.org/10.5326/JAAHA-MS-6999

Fontelles, M. J., Simões, M. G., Farias, S. H., \& Fontelles, R. G. S. (2009). Metodologia da pesquisa científica: diretrizes para a elaboração de um protocolo de pesquisa. Revista paraense de medicina, 23(3), 1-8.

Geiger, R (2021). Classificação climática de Köppen-Geiger. In: Wikipédia, a enciclopédia livre. Fórida: Wikipédia Foundation. Retrieved from https://pt.wikipedia.org/w/index.php?title=Classifica\%C3\%A7\%C3\%A3o_clim\%C3\%A1tica_de_K\%C3\%B6ppen-Geiger\&oldid=60408366

Gide, A. (1967). Métodos adjuvantes na manutenção da temperatura corporal em cães na rotina cirúrgica. Angewandte Chemie International Edition, 6(11), 951-952., 5-24.

Goleman, D., Boyatzis, R., \& Mckee, A. (2019). Castração Pré-Púbere Em Cães E Gatos Benefícios E Riscos. Journal of Chemical Information and Modeling, 53(9), 1689-1699.

Grubb, T., Sager, J., Gaynor, J. S., Montgomery, E., Parker, J. A., Shafford, H., \& Tearney, C. (2020). 2020 AAHA Anesthesia and Monitoring Guidelines for Dogs and Cats. Journal of the American Animal Hospital Association, 56(2), 59-82. https://doi.org/10.5326/JAAHA-MS-7055

Howe L. M. (2006). Surgical methods of contraception and sterilization. Theriogenology, 66(3), 500-509. https://doi.org/10.1016/j.theriogenology.2006.04.005

IBGE - Instituto Brasileiro de Geografia e Estatística. Resultados dos Dados Preliminares do Censo. Portal do IBGE. (2019). <ibge.gov.br>.

João M. da C. N., et al (2011). Control de hipotermia con colchón térmico en perras durante ovario-histerectomía. Revista de Medicina Veterinaria, (22), 11.

Junior, E. D. M. (2015). Causas E Consequencias Da Hipotermia: a Importância Da Monitoração Da Temperatura. 14(6), 1-7.

Luz, M., Münnich, A., \& Vannucchi, C. (2015). Novos enfoques na distocia em cadelas. Revista Brasileira de Reprodução Animal, 39(3), 354-361.

Medina, H. "Como calcular a idade real dos cachorros", 2018. <https://tudosobrecachorros.com.br/idade-canina-X-idade-humana/> 23.01.2021.

Oliveira Filho, K. S., Batista, C. C. N., \& Santos, G. D. dos. (2020). Holidays At the Zoo: Knowing To Preserve. Environmental Smoke, 3(1), 095-099. https://doi.org/10.32435/envsmoke.202031095-099

Pelzer, K., Stebbins, J. F., Prinz, F. B., Borisov, A. S., Hazendonk, P., Hayes, P. G., \& Society, C. (2017). Anestesia em cães e gatos neonatos - Revisão de Literatura. Solid State Ionics, 2(1), 1-10. http://linkinghub.elsevier.com/retrieve/pii/S0167273817305726\%0Ahttp://dx.doi.org/10.1038/s41467017017721\%0Ahttp://www.ing.unitn.it/ luttero/laboratoriomateriali/RietveldRefinements.pdf\%0Ahttp://www.intechopen.com/books/spectroscopic-analysesdevelopments-an

Pottie, R. G., Dart, C. M., Perkins, N. R., \& Hodgson, D. R. (2007). Effect of hypothermia on recovery from general anaesthesia in the dog. Australian Veterinary Journal, 85(4), 158-162. https://doi.org/10.1111/j.1751-0813.2007.00128.x

Radlinsky, M. G. (2015). Cirurgia do Fígado. Cirurgia de Pequenos Animais, 1643-1738. Retrieved from https://www.dropbox.com/home/aulas facul/3 patologia cirurgica II - andrea e rodrigo?preview=Fossum.Cirurgia+de+Pequenos+Animais_+4 + +Edição-ilovepdf-compressed.pdf

Redondo, J. I., Suesta, P., Serra, I. et al. Retrospective study of the prevalence of postanaesthetic hypothermia in dogs, The Veterinary Record, 171(15), 374, 2012. (Redondo, J. I., Suesta, P., Serra, I., Soler, C., Soler, G., Gil, L., \& Gómez-Villamandos, R. J. (2012). Retrospective study of the prevalence of postanaesthetic hypothermia in dogs. The Veterinary record, 171(15), 374. https://doi.org/10.1136/vr.100476)

Rodrigues, N. M., Quessada, A. M., Moraes, A. C., Dantas, S. S. B., \& Sales, K. de K. da S. (2017). Estado físico e risco anestésico em cães e gatos: Revisão. Pubvet, 11(8), 781-788. https://doi.org/10.22256/pubvet.v11n8.781-788

Root Kustritz, M. V. (2014). Pros, cons, and techniques of pediatric neutering. Veterinary Clinics of North America - Small Animal Practice, 44(2), 221-233. https://doi.org/10.1016/j.cvsm.2013.10.002

Van Goethem, B., Schaefers-Okkens, A., \& Kirpensteijn, J. (2006). Making a rational choice between ovariectomy and ovariohysterectomy in the dog: A discussion of the benefits of either technique. Veterinary Surgery, 35(2), 136-143. https://doi.org/10.1111/j.1532-950X.2006.00124.x

Viana, D. C. (2014). Clínica de Pequenos Animais Técnica de ovario-salpingo-histerectomia, em felinos da espécie Felis catus ( gato doméstico ), sem o uso de fios ou lacres. (March 2013). 